\title{
Le prétendu wampum offert à Champlain et l'interprétation des objets muséifiés
}

\section{Jonathan C. Lainey}

Volume 61, numéro 3-4, hiver-printemps 2008

Dossier Québec

URI : https://id.erudit.org/iderudit/019125ar

DOI : https://doi.org/10.7202/019125ar

Aller au sommaire du numéro

\section{Éditeur(s)}

Institut d'histoire de l'Amérique française

ISSN

0035-2357 (imprimé)

1492-1383 (numérique)

Découvrir la revue

Citer cet article

Lainey, J. C. (2008). Le prétendu wampum offert à Champlain et l'interprétation des objets muséifiés. Revue d'histoire de l'Amérique française, 61(3-4), 397-424. https://doi.org/10.7202/019125ar
Résumé de l'article

Outre son fameux astrolabe ou encore son légendaire tombeau, d'autres objets et lieux ont été associés à Samuel de Champlain. C'est le cas d'un des wampums conservés au musée du quai Branly à Paris ayant fait partie de la récente exposition " Premières Nations, Collections royales de France » de passage au Musée Pointe-à-Callière de Montréal à l'été 2007. Assez bien connu du public pour avoir paru dans diverses publications et expositions depuis les cent dernières années, on a affirmé jusqu'à récemment que ce wampum avait été donné par les Hurons à Champlain lui-même en 1611 afin de forger une alliance qui allait garantir le développement de la Nouvelle-France. Bien qu'il eût été intéressant de détenir une " trace " de cette alliance fondatrice, l'apparence des perles et du wampum en général, le silence de Champlain à son égard, la constitution des collections royales et la façon dont cette interprétation erronée s'est développée nous font croire que Champlain n’a jamais vu ni touché ce wampum. Tout en appelant les chercheurs à questionner les objets avec la même rigueur appliquée aux documents écrits, la réflexion entourant un objet en particulier permettra du même coup de parler de la difficulté de documenter et d'interpréter les objets amérindiens muséifiés.
Tous droits réservés @ Institut d'histoire de l'Amérique française, 2008
Ce document est protégé par la loi sur le droit d'auteur. L'utilisation des services d'Érudit (y compris la reproduction) est assujettie à sa politique d'utilisation que vous pouvez consulter en ligne.

https://apropos.erudit.org/fr/usagers/politique-dutilisation/ 


\title{
Le prétendu wampum offert à Champlain et l'interprétation des objets muséifiés
}

\author{
JONATHAN C. LAINEY ${ }^{\mathrm{I}}$ \\ Bibliothèque et Archives Canada
}

RÉSUMÉ • Outre son fameux astrolabe ou encore son légendaire tombeau, d'autres objets et lieux ont été associés à Samuel de Champlain. C'est le cas d'un des wampums conservés au musée du quai Branly à Paris ayant fait partie de la récente exposition «Premières Nations, Collections royales de France » de passage au Musée Pointe-à-Callière de Montréal à l'été 2007. Assez bien connu du public pour avoir paru dans diverses publications et expositions depuis les cent dernières années, on a affirmé jusqu'à récemment que ce wampum avait été donné par les Hurons à Champlain lui-même en 1611 afin de forger une alliance qui allait garantir le développement de la Nouvelle-France. Bien qu'il eût été intéressant de détenir une «trace» de cette alliance fondatrice, l'apparence des perles et du wampum en général, le silence de Champlain à son égard, la constitution des collections royales et la façon dont cette interprétation erronée s'est développée nous font croire que Champlain n'a jamais vu ni touché ce wampum. Tout en appelant les chercheurs à questionner les objets avec la même rigueur appliquée aux documents écrits, la réflexion entourant un objet en particulier permettra du même coup de parler de la difficulté de documenter et d'interpréter les objets amérindiens muséifiés.

1. Je dois remercier Marshall Joseph Becker avec qui je partage, au cours de nos fréquents et réguliers échanges, ma passion pour ces recherches depuis quelques années déjà, Louise Pothier de Pointe-à-Callière, musée d'archéologie et d'histoire de Montréal pour sa collaboration et pour l'accès aux objets, Marie-Paule Robitaille du Musée de la civilisation du Québec pour avoir gentiment fourni ses réflexions et références sur ce wampum bien connu, André Sanfaçon pour avoir accepté de partager le fruit de ses recherches sur les colliers dévotionnels, Normand Laplante, de Bibliothèque et Archives Canada, pour son encouragement et pour avoir facilité la réalisation de cette recherche, et Marie-Claude Nicol pour son support indéniable. Bien entendu, je demeure seul responsable de toute erreur pouvant apparaître dans ces lignes. 
ABSTRACT - Aside from his famous astrolabe and his legendary tomb, other objects and places have been associated with Samuel de Champlain. Such is the case with the wampum band held in the collections of the Quai Branly Museum in Paris, which was part of the recent exhibition titled «First Nations, Royal Collections of France». The exhibition visited the Pointe-à-Callière Museum in Montreal during the summer of 2007. Fairly well known for having appeared in various publications and exhibitions over the last century, until recently it was claimed that this wampum had been given to Champlain himself by the Hurons in 1611, in order to forge an alliance which would ensure the development of New France. As interesting as it would have been to identify this founding alliance, the appearance of the individual beads and of the wampum band in general, Champlain's silence on the subject, the constitution of the royal collections and the way in which this mistaken interpretation developed makes it clear that Champlain never saw or touched this wampum. While underscoring the need for researchers to question objects with the same rigour they apply to written documents, a reflection on this particular object also provides a context for discussing the difficulty of documenting and interpreting Amerindian objects which have been integrated into museum collections.

\section{INTRODUCTION}

Je crois que les gens souhaitent que ça soit vrai parce que nous avons besoin de ces choses pour construire notre histoire. [...]

C'est fantastique d'être capable de lui toucher et de dire: "Ceci fut utilisé par Champlain²."

Dans les journaux canadiens couvrant l'ouverture officielle du musée du quai Branly à Paris, qui eut lieu au mois de juin 2006, on annonce en grande pompe que le nouveau musée est dépositaire d'un collier de wampum offert à Champlain lui-même, témoin tangible exceptionnel des relations et alliances entre Amérindiens et Français ${ }^{3}$. Le collier de wampum qui nous intéresse mesure $83 \mathrm{~cm}$ (hors frange) pour une largeur de $12 \mathrm{~cm}$,

2. Jean-Pierre Chrestien, conservateur, archéologie du Québec, au Musée canadien des civilisations, à propos de l'astrolabe dit de Champlain, cité dans Paul Gessell, «The "star-taker" », The Ottawa Citizen, 10 mai 2006, A6. Traduction libre de l'auteur.

3. «Native Art Part of New Paris Museum: Artifacts Given to Samuel de Champlain Part of Non-Western Culture Exhibition", Times Colonist, 21 juin 2006, C12; "Canadian Artifacts on Display in the Shadow of the Eiffel Tower: New Paris Museum Draws Praise and Criticism for Exhibiting the Spoils of Colonialism», The Ottawa Citizen, 21 juin 2006, A3; «Museum Features Aboriginal Artifacts», Edmonton Journal, 21 juin 2006, A4; «Native Art Displayed in Paris», The Windsor Star, 21 juin 2006, C10 ; «A New Take on "The Spex of Culture”: Non-Western Cultures, Including Canadian Aboriginals, Will Be the Focus of Art at Quai Branly Museum in Paris", Montreal Gazette, 29 juin 2006, A21. 
il comprend dix-sept rangs de perles de coquillages de large et, au musée du quai Branly, il porte le numéro 71.1878.32.61. Quatre figures humaines blanches tournées alternativement vers la gauche et vers la droite et tenant ce qui ressemble à des arcs (certains y voient des boucliers) sont illustrées sur un fond de perles pourpres. Le prétendu wampum offert à Champlain est assez connu du public en général pour avoir fait l'objet de publications et expositions diverses, notamment avec la collaboration d'institutions canadiennes. Il fut interprété quelque peu différemment à travers le temps, avec plus ou moins d'information selon les auteurs. Jusqu'à tout récemment, l'interprétation véhiculée contenait les informations suivantes: «Wampum dit des quatre nations huronnes qui aurait été donné à Champlain en 1611 par Atironta, un chef des Arendahronnons (une des quatre nations de la confédération huronne), dans le but de demander alliance à la France.»

Or, en enquêtant davantage, on se rend compte rapidement que rien ne permet de corroborer cette affirmation étonnante. Ainsi, nous argumenterons que cette interprétation n'est pas valide et expliquerons pourquoi toute tentative d'en proposer une autre s'avère pratiquement vaine. Après avoir présenté brièvement ce qu'est le wampum, nous tenterons de retracer l'origine de cette interprétation erronée pour enfin discuter de la possibilité de documenter et d'interpréter ce genre d'objets muséifiés. En ce sens, le présent article souhaite proposer des éléments et aspects à considérer lorsqu'il s'agit d'étudier un wampum. En effet, les critères de base servant à l'étude de l'objet matériel, souvent, ne s'appliquent pas. Plusieurs informations pourtant fondamentales à l'étude de l'objet ne peuvent être fournies (d'où vient-il? qui l'a produit? dans quel contexte fut-il utilisé? quand? par qui? etc. $)^{4}$. Bien sûr, une première observation de visu permet de dire assez aisément de quoi il s'agit: un collier de porcelaine. Mais le sens de l'objet et sa signification demeurent toutefois insondables puisque le wampum, nous le verrons, devait parfois, à l'origine, signifier quelque chose en particulier. Ces importantes contraintes documentaires nous conduisent à privilégier d'autres aspects qui sont tout de même reliés aux objets et qui permettent de mieux les comprendre.

4. Voir, entre autres, Jacques Mathieu, "Comment analyser l'objet matériel», dans Jocelyn Létourneau, Le coffre à outils du chercheur débutant. Guide d'initiation au travail intellectuel (Toronto, Oxford University Press, 1989), 93-100. Version écourtée de Jacques Mathieu, avec la participation de Georges-Pierre Léonidoff et John R. Porter, «Comment analyser l'objet matériel», Material History Bulletin/Bulletin d'histoire de la culture matérielle, 26 (1987): 7-18; Joan M. Vastokas, «Native Art as Art History: Meaning and Time from Unwritten Sources», Journal of Canadian Studies/ Revue d'études canadiennes, 21,4, (hiver 1986-1987): 7-36. 
C'est ce que nous entendons faire dans l'étude de cas que nous proposons.

De façon parallèle, notre réflexion nous permettra d'aborder la délicate question de l'interprétation des objets dans les musées. En effet, traiter du sens rattaché à un objet en particulier permettra du coup d'interroger nos capacités à interpréter la culture matérielle, tout comme la façon dont celle-ci peut être parfois mise au service d'un discours identitaire rassembleur. Les musées qui conservent les précieux objets issus du passé sont des lieux de constitution et de préservation de collections, mais ils sont aussi des lieux de communication et de partage de savoirs et de mémoires. L'interprétation des objets est donc l'un des aspects les plus fondamentaux du travail des musées. Lorsqu'on sait que ces institutions demeurent pour le public un point de contact privilégié avec l'histoire, et lorsqu'on considère que les musées représentent pour plusieurs une source excessivement fiable et crédible pour comprendre le passé, on est en droit de se demander s'il faut à tout prix offrir au public une interprétation «quelle qu'elle soit $^{5} »$.

\section{QUELQUES MOTS SUR L'EXPRESSION WAMPUM(S)}

Plus d'un siècle de littérature scientifique et amateure sur les wampums prouve que beaucoup de choses peuvent être dites sur ces objets. Dans le cadre de cet article, nous nous contenterons d'insister sur les aspects que nous jugeons importants et qui sont pertinents à notre propos. Le mot wampum est une abréviation de wampumpeague ou wampumpeake, un mot algonquin du sud de la Nouvelle-Angleterre récupéré par la langue anglaise au début du $\mathrm{XvII}^{\mathrm{e}}$ siècle et qui signifierait tout simplement «enfilade de perles de coquillages blancs ${ }^{6} »$. Bien que le terme wampum fut souvent utilisé pour désigner différents types de perles de coquillages ou d'objets perlés, il réfère à un type de perles en particulier. Celles-ci se distinguent des autres par la combinaison de quatre critères: le matériel utilisé (coquillages marins provenant exclusivement des côtes de l'océan Atlantique), leur forme tubulaire, leur taille (environ 3 à $5 \mathrm{~mm}$ de diamètre sur 7 à $10 \mathrm{~mm}$ de longueur) et leur technique de fabrication (per-

5. L'expression est de Cheryl Mezsaros, dans "Sous le règne de l'interprétation", Muse (janvier-février 2007): 24-29. Sur le rôle des musées comme lien privilégié avec le passé, voir Roy Rosenzweig et David Thelen, The Presence of the Past. Popular Uses of History in American Life (New York, Columbia University Press, 1998).

6. J. N. B. Hewitt, "Wampum», dans Frederic Webb Hodge, dir., Manuel des Indiens du Canada (Ottawa, J. de L. Taché, 1915 [1913]), 619. 
çage et polissage) qui nécessitait des outils de métal d'origine européenne ${ }^{7}$.

Vers 1620-1625, l'utilisation grandissante de pointes de métal fit en sorte d'accroître considérablement la fabrication des perles tubulaires standardisées et uniformisées qui se répandirent à l'intérieur du continent en devenant un bien d'échange essentiel à l'immense réseau de commerce qui s'étendit pendant la période de la traite des fourrures. Des manufactures de perles de wampum seront établies par les colons hollandais le long des côtes pour tenter de faire compétition aux producteurs amérindiens, Narragansetts et Péquots notamment ${ }^{8}$. Ces peuples algonquiens utilisèrent les perles pour s'orner et participer à l'économie de la traite des fourrures, tandis que les Hollandais, et les Anglais dans une moindre mesure, les utilisèrent pendant un certain temps comme une forme de monnaie à défaut d'avoir accès à des pièces de monnaie métalliques.

Les peuples iroquoiens de l'intérieur des terres, quant à eux, firent un usage tout particulier du wampum en l'utilisant dans leurs rencontres diplomatiques officielles avec les groupes voisins, Amérindiens ou Européens. Ces perles étaient alors tissées en des colliers de diverses tailles pouvant contenir de quelques centaines à plus de dix mille perles. Par l'alternance des couleurs des perles, blanches et pourpres uniquement, des motifs étaient généralement illustrés (carrés, losanges, hexagones, triangles, croix, lignes parallèles ou obliques, zigzags, cercles, pipes, haches, bâtiments, figures animales ou humaines, écritures, chiffres). Ces colliers étaient offerts lors des rencontres diplomatiques officielles pour supporter et rendre légitime le discours prononcé. Les wampums échangés étaient parfois conservés sur de longues périodes et leur "contenu » devait être expliqué régulièrement afin que l'on se remémore la teneur des ententes passées. L'échange de wampums se réalisait selon des règles protocolaires précises et particulières que tous les acteurs en présence se devaient de respecter ${ }^{9}$. On retrouvait ce système diplomatique plus spécifiquement au sein des trois confédérations iroquoiennes du Nord-Est américain, soit les Iroquois, les Andastes (Susquehannock en anglais) et

7. Lynn Ceci, «The First Fiscal Crisis in New York», Economic Development and Cultural Change, 28,4, (juillet 1980): 839-847.

8. Ibid.

9. Mary A. Druke, «Iroquois Treaties, Common Forms, Varying Interpretations», dans Francis Jennings, dir., The History and Culture of Iroquois Diplomacy. An Interdisciplinary Guide to the Treaties of the Six Nations and Their League (New York, Syracuse University Press, 1985), 92-96; Jonathan C. Lainey, La "monnaie des Sauvages». Les colliers de wampum d'hier à aujourd'hui (Sillery, Septentrion, 2004), 38-49. 
les Hurons. Il semble aussi qu'après leur dispersion vers 1650, ces derniers auraient transmis et diffusé l'usage du wampum en diplomatie dans la vallée du Saint-Laurent, et dans la région des Grands Lacs ${ }^{10}$.

L'espace géopolitique et culturel des Pays d'en haut (région des Grands Lacs, incluant le haut du Mississippi $)^{11}$ serait aussi inclus, du moins partiellement, dans ce que l'on pourrait nommer le "cœur» ou le "noyau» de cette tradition où l'usage des wampums en diplomatie est essentiel. La région du «centre» se distingue de la "périphérie» où l'usage des wampums en diplomatie est rare sinon absent. En effet, les travaux de Marshall J. Becker montrent que les peuples algonquiens côtiers n'utilisaient pas les colliers de wampum entre eux, dans leurs relations diplomatiques, et ce, bien que les peuples voisins plus au nord et à l'ouest leur en aient offerts et leur en aient présentés à l'occasion ${ }^{12}$. Bien que jusqu'au milieu du XvII ${ }^{\mathrm{e}}$ siècle la "périphérie» ait inclus aussi la vallée du SaintLaurent ${ }^{13}$, il est probable que certains groupes d'Algonquins occupant le nord du fleuve et la vallée de l'Outaouais étaient déjà familiers avec l'usage des wampums en diplomatie au début du XviI siècle, compte tenu de leur proximité particulière avec les Hurons. Bref, même si la tradition d'échange des wampums s'est répandue dans le Nord-Est américain, de la vaste région des Grands Lacs jusque dans les Maritimes, il est clair que tous les groupes en présence, Européens inclus, n’utilisèrent pas le wampum de la même manière. Le développement de l'usage des wampums dans le cadre de relations internationales se réalisa donc dans un espace spéci-

10. Gilles Havard, Empire et métissages. Indiens et Français dans les Pays d'en Haut, 1660-1715 (Sillery/Paris, Septentrion/Presses de l’Université de Paris-Sorbonne, 2003), 173, 201 note 243.

11. Ibid., 11-14.

12. Marshall Joseph Becker, «Wampum Held by The Oneida Indian Nation, Inc. of New York: Research Relating to Wampum Cuffs and Belts", Bulletin of the New York State Archaeological Association, 123 (2008), à paraître.

13. Des exemples tirés des Relations des Jésuites le suggèrent: le 2 juillet 1636, Montagnais et Algonquins donnent des peaux aux Français pour accompagner leur discours; Reuben Gold Thwaites, dir., The Jesuit Relations and Allied Documents: Travels and Explorations of the Jesuit Missionaries in New France, 1610-1791 (New York, Pageant Book Company, 1959 [1896-1901]), IX: 226-232. En 1643-1644, des Algonquins leur remettent encore des peaux de castor; Ibid., XXV: 254-268. En juin 1642 et en juillet 1645, les Français reçoivent des colliers de porcelaine de la part des Iroquois; Ibid., XXI : 42-46; Ibid, XXVII : 246-272. Quelque temps après la dispersion des Hurons, soit en 1653, des Algonquins offrent des colliers de porcelaine aux Abénaquis; Ibid., XL: 202-208. En d'autres termes, le don de présents demeure à la base de la pratique diplomatique, mais les Hurons et Iroquois, pour des raisons géopolitiques et commerciales, offrent des colliers de wampum. À noter que dans l'une de ses excellentes études sur les colliers de porcelaine parues en 1970 et 1971, André Vachon avait déjà remarqué ces distinctions; André Vachon, "Colliers et ceintures de porcelaine chez les Indiens de la Nouvelle-France», Cahier des Dix, 35 (1970): 270. 
fique, dans la région du "centre», là où le contact s'est effectué avec une grande intensité, avec beaucoup de diplomatie et de jeux politiques.

Il importe enfin de faire une distinction entre «le» wampum et «les» wampums. Lorsqu'on parle «du» wampum, on renvoie au matériel, à la matière première, c'est-à-dire aux perles. Par contre, lorsqu'on parle «des » wampums, on réfère alors aux colliers de wampum (ou de porcelaine), qui sont en fait des bandes perlées de plusieurs rangs de perles tissées. Les Français nommeront les wampums «colliers de porcelaine» et les Anglais «wampum belts». Ces deux expressions identifient le même objet échangé lors des rencontres diplomatiques officielles ${ }^{14}$. Enfin, il faut distinguer un collier d'une «branche de porcelaine» (en anglais string of wampum), cette dernière étant constituée de perles enfilées sur une corde seulement. On échangeait les branches isolément ou attachées ensemble pour former une unité de plusieurs centaines de perles.

\section{LE WAMPUM DIT DES QUATRE NATIONS HURONNES}

Pour commencer l'analyse du wampum qui nous intéresse, attardonsnous d'emblée à ses caractéristiques matérielles en insistant sur trois aspects en particulier: l'apparence des perles, la taille du wampum et les motifs qui y sont illustrés. En observant l'objet de près, une chose frappe d'abord: les perles utilisées sont étonnement fines et uniformes. Or, ces caractéristiques sont généralement celles de perles fabriquées de façon «industrielle» avec des outils européens dans les manufactures établies à cette fin, à partir de 1615-1620 environ ${ }^{15}$. En effet, la traite de cet article venait tout juste de débuter dans l'Est du continent, dans les colonies hollandaises notamment, où s'installe de façon permanente en 1614 la Nieuw Nederland Compagnie nouvellement formée ${ }^{16}$. La fabrication et la présentation de colliers et de branches de wampum dans les conseils intertribaux se sont développées sensiblement à cette période, et les

14. Dans la littérature sur les wampums, on retrouve parfois l'utilisation de l'expression «ceinture de porcelaine», qui n'est en fait que la traduction de l'anglais « wampum belt». Pourtant, dans les archives françaises, les wampums sont toujours désignés par «colliers de porcelaine» et les Français échangent de ces colliers de la même façon que les Anglais échangent des «wampum belts». Certaines traductions anglaises de sources françaises ont souvent traduit le mot collier par "collar», ce qui porte davantage à confusion.

15. William C. Orchard, Beads and Beadwork of the American Indians (New York, Museum of the American Indian, Heye Foundation, Contributions from the Museum n 11,1929$)$; Frank G. Speck et W. C. Orchard, The Penn Wampum Belts (New York, Museum of the American Indian, Heye Foundation, leaflet $\left.n^{\circ} 4,1925\right)$; E. B. Tylor, «Notes and Addenda: The Hale Series of Wampum Belts ", Journal of the Royal Anthropological Institute, 26 (1897): 248-254.

16. L. Ceci, "The First Fiscal Crisis...», loc. cit., 842. 
colliers diplomatiques que l'on connaît furent utilisés plus couramment en Huronie seulement vers 1630, bien que des branches de perles de coquillages puissent l'avoir été dès le début du xvir siècle.

Ainsi ce que remarquent Marc Lescarbot en Acadie en 1606-1607 et Champlain à Cape Cod en 1606 et ce que reçoit Henry Hudson des Mahicans en 1609 sont davantage des "branches» que des "colliers» tels qu'on les conçoit, ou comme Gabriel Sagard les décrit en Huronie en 1623-1624 («larges de trois ou quatre doigts, [...] et [...] environ trois pieds et demi de tour ou plus $\left.{ }^{17} »\right)$. Les mentions de wampum dans les écrits de Champlain montrent qu'il y avait bien du wampum en Huronie avant 1615, mais pas en quantité importante. On peut imaginer que les perles plus régulières ont été fabriquées à partir des outils plus performants, plus sophistiqués, donc plus tardifs. Il faut dès lors s'interroger sur la présence de ce type de perles en Huronie au tout début du XviI ${ }^{\mathrm{e}}$ siècle. Les Hurons avaient-ils accès à des perles standardisées et uniformisées en quantité suffisante pour offrir un collier de la sorte à Champlain? On peut en douter fortement.

D'autres critères font en sorte que l'âge prétendu du wampum est problématique: soit sa taille et les motifs qu'il comporte. Sans pour autant que cet aspect puisse être jugé déterminant pour situer l'objet dans le temps, il est généralement admis que les wampums comportant des motifs dits "complexes» sont moins anciens que ceux comprenant des motifs très simples, tels des lignes obliques ou des losanges. Ce serait dans la deuxième partie du $\mathrm{xvIII}^{\mathrm{e}}$ siècle seulement que des wampums de grande taille et aux motifs plus élaborés furent présentés de façon plus soutenue ${ }^{18}$. Justement, la taille du wampum ici étudié est somme toute considérable. Il comporte dix-sept rangs de large, pour un total d'environ 3800 perles $^{19}$.

17. André Vachon supposait qu'il s'agissait déjà de "colliers» de wampum; A. Vachon, "Colliers et ceintures de porcelaine.... loc. cit., 253-255.

18. William M. Beauchamp, Wampum and Shell Articles Used by the New York Indians (New York, AMS Press, 1978 [1901]), 391-403; M. J. Becker, «A Wampum Belt Chronology: Origins to Modern Times", Northeast Anthropology, 63 (printemps 2002) : 58-59, 62-63; Michael K. Foster, "Another Look at the Function of Wampum in Iroquois-White Councils », dans F. Jennings, dir., The History and Culture of Iroquois Diplomacy..., op. cit., 123.

19. Par exemple, en 1748, alors que le wampum est abondant et couramment utilisé dans le Nord-Est, un wampum "standard» comportait environ mille perles, tandis qu'un «large» wampum en comportait plus du double. En guise d'exemple, voir le décompte que fait Conrad Weiser des wampums qu'il a reçus pendant les deux rencontres auxquelles il a assisté dans Samuel Hazard, dir., Pennsylvania Archives (Philadelphia, Joseph Severns and Company, 1852) [1 ${ }^{\text {re }}$ série], II : 17. Voir aussi l'«Estat de la depense qui a été faite à Montréal» en août 1746 dans lequel on dénombre « 2800 Grains de porcelaine En un Grand Collier» «délivré pour presenter le Casseteste aux hurons, Outaouais, Sauteus, poutéatomis du détroit»; Bibliothèque et Archives Canada, MG1, 
Il serait douteux que les Hurons aient offert aux Français une telle quantité de perles de wampum par ailleurs peu nombreuses en Huronie.

L'état général du wampum est pratiquement impeccable, le tissage semble toujours très solide et quatre perles seulement sont manquantes (deux à l'extrémité du haut, une autre tout juste en haut de l'arc du deuxième personnage et une dernière en haut du bras tenant l'arc du personnage du bas). Les wampums ne sont pas toujours en si bon état. Plusieurs sont brisés, les fils cassés, plusieurs perles sont manquantes ou même une partie entière du wampum peut être détachée ${ }^{20}$. Et pourtant, daté de 1611, il serait l'un des plus vieux wampums connus.

Comparons-le avec un autre wampum de la collection du musée du quai Branly, aussi exposé au Musée Pointe-à-Callière à l'été 2007, sur

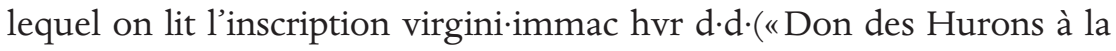
Vierge Immaculée»). Les conclusions les plus récentes sur les wampums comportant des écritures latines suggèrent que ce wampum aurait été fabriqué en 1666 par les Hurons alors établis dans un fort construit dans la ville de Québec ${ }^{21}$. Ce wampum est constitué de perles de formes et de tailles beaucoup moins régulières, plusieurs sont d'ailleurs manquantes et comme il est très vieux, son état général laisse à désirer.

Précisons que l'analyse et la comparaison des perles, du tissage et de l'état du wampum ne sont absolument pas garantes d'une datation précise. Bien sûr, le contexte dans lequel l’objet est préservé est primordial quant à son état de conservation. L'on sait aussi que des perles et des colliers de wampum ont été fabriqués et échangés pendant pratiquement deux siècles, et que les perles d'un wampum défait pouvaient être réutilisées pour la fabrication d'autres wampums. Dater les perles ne permet donc pas de dater l'objet lui-même puisque celui-ci peut être constitué de perles de diverses provenances ${ }^{22}$. Néanmoins, en fonction de la date qui est proposée (1611), ces critères demeurent d'une certaine utilité, du

C11A. Fonds des colonies. Correspondance générale; Canada (1540-1784), vol. 115, «Estat de la depense qui a été faite à Montréal pour et a l'Occasion du party de françois et Sauvages Commandé par M. Rigaud de Vaudreuil major de la place du Trois Rivières pour aller sur les Costes de la Nouvelle Angleterre party de Cette Ville le Trois Aoust 1746», par Jean-BaptisteGrégoire Martel de Saint-Antoine, Montréal, 30 septembre 1746, folio 266, bobine C-2407.

20. Voir des images dans W. M. Beauchamp, Wampum and Shell Articles..., op. cit. et dans Francis Jennings, dir., Iroquois Indians: A Documentary History of the Diplomacy of the Six-Nations and Their League. The Microfilm Collection (Connecticut et Angleterre, Woodbridge et Reading, The D'Arcy McNickle Center for the American Indian, The Newberry Library, 1984), bobine 50.

21. André Sanfaçon, Wampums chrétiens. Offrandes de colliers de porcelaine par les missions et réductions jésuites de la Nouvelle-France, à paraître.

22. J. Lainey, La "monnaie des Sauvages»..., op. cit., 76-78, 201-203. 
moins pour évacuer le tout début de la période de production de perles et de leur utilisation dans le réseau diplomatique.

\section{LE SILENCE DE CHAMPLAIN}

Voyons maintenant ce que Champlain rapporte de sa rencontre avec les Hurons en 1611 et des présents qu'il reçoit à cette occasion. Aux premiers abords, il semble que l'objet qui nous intéresse ne figure pas dans les écrits de Champlain. Il est vrai toutefois que celui-ci reçoit des «colliers» de coquillages en 1611 de la part des Hurons afin de consolider une première alliance survenue en 1609 , et c'est sans doute pour cette raison que l'on a voulu associer l'objet avec cet événement précis. En juin 1611, environ deux cents Hurons et Algonquins se rendirent aux rapides de Lachine pour rencontrer Champlain et Gravé du Pont et leur remettre en secret: « 50 castors $\& 4$ carquans de leurs porcelaines (qu'ils estiment entre eux comme nous faisons les chaisnes d'or $)^{23}{ }^{\prime}$. On expliqua aux Français que ces présents provenaient des chefs hurons qui étaient restés en Huronie et qui désiraient fortement établir des liens avec eux.

Il est difficile de juger si, en 1611, les perles offertes se qualifient pour être du «vrai» wampum comportant les caractéristiques nommées cihaut, tel qu'il fut produit quatre ou cinq ans plus tard dans la sphère de traite hollandaise, tout comme il est difficile de savoir si ces "carcans" renvoient bien à des colliers de wampum tels qu'on les connaît. Il est possible que ce ne soit pas le cas, bien qu'il faille reconnaitre que les objets furent effectivement offerts dans un contexte diplomatique. En effet, le mot «carquan » renvoie à « une espèce de chaisne d'orfevrerie, ou de pierreries $^{24}$ », ce qui est probablement différent des «colliers de pourceline», tels que Champlain les nomme, pour la première fois en 1616, alors que les "Algommequins» en offrent cinquante, entre autres objets, aux "Atignouaatitans» (les Attignaouantans étant la nation de l'Ours, l’une des nations de la confédération huronne $)^{25}$.

23. Georges-Émile Giguère, dir., Euvres de Champlain (Montréal, Éditions du Jour, 1973), I: 402 et II : 849.

24. Dans la première édition du Dictionnaire de L'Académie française (1694), on peut lire la définition suivante : "CARCAN. s. m. Cercle de fer avec lequel on attache un criminel par le cou à un poteau. Condamner au carcan. mettre au carcan. attacher au carcan. Il sign. aussi, Une espece de chaisne d'orfevrerie, ou de pierreries, mais il n'est plus guere en usage en ce sens-là ». Le Thresor de la langue française (1606) de Jean Nicot ne donne rien pour «carquan» ou "carcan». (Dictionnaires consultés le 11 juillet 2008 à l'adresse Internet suivante: http://www.lib.uchicago.edu/efts/ ARTFL/projects/dicos/).

25. G.-É. Giguère, dir., Euvres de Champlain..., op. cit., II : 550. 
L'analyse du texte proprement dit suggère que Champlain reçut seulement des branches de perles de coquillages, possiblement faites de perles de wampum. Bien que certains aient suggéré que chacun de ces «carquans " aurait représenté une des quatre nations huronnes et que chacune des cinquante peaux aurait représenté un des chefs hurons ${ }^{26}$, cela demeure de loin insuffisant pour être en mesure de relier l'objet du musée du quai Branly avec cet événement précis. Surtout si l'on considère que l'objet de près de quatre mille perles qui nous intéresse ici ne représenterait qu'un seul de ces quatre "carquans». Si Champlain avait effectivement reçu un tel objet, il est probable qu'il en aurait parlé plus longuement, au moins en en faisant une description sommaire. La description des objets échangés figure justement parmi les informations les plus importantes et c'est précisément cette information qui fait cruellement défaut aux chercheurs qui souhaitent relier les wampums aux documents d'archives. Sans une description exhaustive des objets échangés, toute association à des événements historiques devient hypothétique et hautement spéculative.

\section{LA CONSTITUTION DES COLLECTIONS ROYALES DE FRANCE ET LEUR DOCUMENTATION AUJOURD'HUI}

Comme le remarquait avec justesse le responsable des collections des Amériques du musée du quai Branly, André Delpuech, lors d’une cérémonie d'accueil impliquant des représentants et Aînés amérindiens au musée Pointe-à-Callière le $1^{\text {er }}$ juin 2007, les collections royales de France sont fort connues du public et des chercheurs, mais en même temps, et bien paradoxalement, elles sont fort méconnues. On sait qu'elles existent, mais on connaît peu leur histoire et leurs origines. Les collections royales de France (tout comme les collections muséologiques en général pourrait-on dire) sont en effet peu documentées du fait qu'elles sont très anciennes, qu'elles proviennent de multiples sources et qu'elles changèrent plusieurs fois de mains au fils du temps.

Pour réaliser l'exposition "Premières Nations, collections royales » tenue au musée du quai Branly de février à mai 2007, le commissaire Christian Feest a eu accès à une dizaine de catalogues et inventaires des collections de "curiosités» qui constituaient autrefois les cabinets des collectionneurs privés ou des institutions publiques. Réalisés à différents moments dans l'histoire sur une période de deux siècles (entre 1670 et 1869), ces catalogues, qui sont absolument nécessaires à l'étude des

26. Bruce G. Trigger, Les Indiens, la fourrure et les Blancs. Français et Amérindiens en Amérique du Nord (Montréal, Boréal, 1992 [1985]), 249. 
collections, dévoilent clairement le manque de documentation des objets. Le mutisme des sources à cet égard est frappant: le silence entourant les pièces, l'anonymat tout comme la diversité des donateurs étonnent et laissent le chercheur perplexe. Quelques pièces seulement peuvent être reliées avec une certaine certitude à des collectionneurs ou à des groupes amérindiens.

Il va sans dire, penser être en mesure de retracer exactement la provenance d'un objet à partir de ces sources relève du domaine de l'utopie. Explorateurs, négociants, navigateurs, missionnaires, fonctionnaires coloniaux, cabinets privés de curiosités ou d'histoire naturelle, musées, couvents, communautés religieuses, maisons des grands seigneurs, châteaux royaux, tous ont contribué, de diverses façons, et ce, dès le début du xviI ${ }^{\mathrm{e}}$ siècle, à constituer les collections ethnographiques que l'on peut admirer aujourd'hui ${ }^{27}$. Il faut aussi admettre que malgré une curiosité sincère et un attrait certain envers les objets exotiques, inédits, "curieux", l'intérêt ethnographique des premiers collectionneurs n’y était pas réellement.

Un document d'archives très pertinent à ce propos l'illustre assez bien. Dans son «Memoire des Curiosités qui me sont venues de Canada» daté du 9 janvier 1689, Michel Bégon est très peu explicite sur la provenance, la description et la documentation des objets qu'il reçoit: «Une paire de brasselets de rassades", "un collier de capitaine avec sa coquille et ses gallons ou canons de porcelaine», "collier de femme ou de fille avec deux paires de pendants doreilles de porcelaine», sont des exemples des descriptions que l'on y retrouve. Sur les quelque soixante objets énumérés, allant du simple spécimen géologique aux objets ethnographiques plus élaborés, sept seulement sont identifiés par leur origine ethnique (deux hurons, un «abnaquie», un «esckomaun(?)» [Eskimo?], deux «outaouac» (ou «oütaoüas»), un «Ilinoise $»^{28}$.

Selon le conservateur Hamy, après avoir été confisqué en 1792 par application de la «loi sur les émigrés» (ces «émigrés» étant essentielle-

27. Sur l'histoire de ces collections et leur cheminement à travers le temps, voir Pascal Riviale, «Les collections ethnographiques d'Amérique du Nord en France dans la seconde moitié du XviII ${ }^{\mathrm{e}}$ siècle», dans Christian F. Feest, dir., Premières nations, collections royales: les Indiens des forêts et des prairies d'Amérique du Nord (Paris, Musée du quai Branly et Réunion des musées nationaux, 2007), 59-64; C. F. Feest, "La collection de curiosités "sauvages" " et "Les collections royales depuis 1800 », dans Ibid., 51-56, 67-73); C. F. Feest, «First Nations - Royal Collections », American Indian Art Magazine, 32,2 (2007): 45-47; Ernest-Théodore Hamy, Les origines du Musée d'ethnographie (Paris, Jean-Michel Place, Cahier de Gradhiva 7, 1988 [1889]), chap. III.

28. Bibliothèque et Archives Canada, MG7, IA2. Département des manuscrits. Fonds français. Collection Dangeau (1656-1719), Lettre signée de Michel Bégon à Cabart de Villermont, 9 janvier 1689 , Rochefort, folios $186-187 \mathrm{v}$, bobine F-486. 
ment les aristocrates qui s'exilaient à l'étranger pour échapper à la justice révolutionnaire, après 1789), le "wampum des Quatre-Nations huronnes" aurait été déposé avec cinq autres dans les collections du Cabinet des Antiques à la Bibliothèque nationale pour ensuite être transféré, vers 1880, au Musée d'ethnographie du Trocadéro ${ }^{29}$. Selon Feest, seul un inventaire de 1796 contient une entrée qui pourrait renvoyer au collier qui nous intéresse; on parle alors d'un "Indian war belt ${ }^{30}$ ». Encore une fois, à partir d'une description aussi sommaire, rien n'est moins sûr. Et ce, d'autant plus que le wampum ne renferme aucun reste de pigment rouge, ce qui aurait pu indiquer un contexte guerrier. En effet, les archives coloniales sont assez explicites sur le sujet: les «colliers de guerre» échangés étaient généralement peints de vermillon ou d'ocre rouge ${ }^{31}$.

Visiblement, étant donné le manque d'informations fiables et exhaustives, et considérant le fait que ces collections royales sont constituées de multiples sources, il devient impossible de retracer l'origine du wampum dit de Champlain. Par contre, il n'est pas impossible qu'un jour nous découvrions d'autres sources qui pourraient nous en dire davantage. $\mathrm{Si}$ les comptes rendus ou procès-verbaux des rencontres officielles contiennent parfois une description sommaire des wampums échangés (surtout après 1740), ne sous-estimons pas l'apport d'autres sources historiques.

En guise d'exemple, nous voudrions mentionner l'existence d'un document d'archives qui semble pertinent pour l'étude de trois objets fabriqués de perles de wampum issus des mêmes collections royales de France, qui furent eux aussi exposés au musée Pointe-à-Callière à l'été 2007: un collier de wampum fendu en plein centre pour y passer la tête et le porter sur les épaules $\left(\mathrm{n}^{\circ} 71.1878 .32 .56\right)$ et une paire de bracelets aussi fabriqués de perles de wampum ( $\left.\mathrm{n}^{\circ} 71.1878 .32 .60\right)$. Il s'agit d'un «Memoire concernant les Coliers de Porcelaine des Sauvages, leurs differents usages et la matière dont ils sont composés » rédigé vers 1726-1727 dans lequel on peut lire:

Les Sauvages se servent aussi de ces Coliers pour leur servir d'ornement en les pendant à leur Col. Ils en font aussi d’autres qui sont fendus par le milieu

29. E.-T. Hamy, «Note sur un wampum représentant les quatre-nations des Hurons», Journal de la Société des américanistes de Paris, 1 (1897):166. À noter que Léo Leymarie propose une autre provenance à ce wampum, le rattachant plutôt au cabinet d'histoire naturelle du marquis de Sérent, à Versailles; Léo Leymarie, Catalogue illustré. Exposition rétrospective des colonies françaises de l'Amérique du Nord. Analyse des documents, objets et peintures exposés (Paris, Société d'éditions géographiques, maritimes et coloniales, 1929), 1. Voir aussi P. Riviale, «Les collections ethnographiques...", loc. cit., 62-64.

30. C. F. Feest, «First Nations - Royal Collections», loc. cit., 55, note 14.

31. J. Lainey, La "monnaie des Sauvages»..., op. cit., 109, 173-174, $2^{\mathrm{e}}$ de couverture. 
pour les passer au Col, ils en font encore des Brasselets larges de 4 pouces au moins qu'ils mettent sur leurs poignets. L'on peut voir de ces brasselets et un de ces coliers dans le Cabinet de M. de Paiot d'Oms-en-Bray. La Porcelaine employée soit en coliers soit en Brasselets est enterrée avec celuy à qui elle appartenoit $^{32}$.

Bien que succinct, ce passage est extrêmement intéressant pour plusieurs raisons. D’abord, il décrit des objets très particuliers dont peu d'équivalents existent dans le monde: on dénombre actuellement treize bracelets de wampum dans diverses collections et seulement un autre collier du genre, passablement brisé, qui aurait été acquis à Akwesasne en $1883^{33}$. Le mémoire décrit aussi une pratique amérindienne qui consiste à enterrer ces objets avec le défunt qui les possédait, ce qui pourrait expliquer le fait que peu d'objets semblables nous soient parvenus. De plus, il donne le nom d'un individu, «M. de Paiot d'Oms-en-Bray», qui apparemment possédait son propre «cabinet de curiosités ${ }^{34}$ ». Or, parmi tous les noms des anciens propriétaires de cabinets qui sont énumérés dans l'ouvrage accompagnant l'exposition «Premières nations, collections royales $^{35}$ ", celui-ci n’apparaît pas. Qui plus est, il est admis que ces trois objets formaient originellement un ensemble du fait notamment que tous trois comportent une bordure de piquants de porc-épic teints en rouge ${ }^{36}$. Dès lors, il est permis de penser que l'auteur anonyme de ce mémoire a bel et bien décrit des objets excessivement rares qu'il a pu lui-même

32. Bibliothèque nationale de France, Paris, Manuscrits occidentaux. Nouvelles acquisitions françaises, vol. 2550, «Memoire concernant les Coliers de Porcelaine des Sauvages, leurs differents usages et la matière dont ils sont composés ", anonyme, n. d. [circa 1726-1727], folio 25. À noter que ce document inédit a été partiellement publié dans le Bulletin des recherches historiques, 55,4-5-6 (1949): 119-120.

33. Marshall J. Becker, «Unique Huron Ornemental Bands: Wampum Cuffs», Material Culture Review/Revue de la culture matérielle, 66 (automne 2007): 60; C. F. Feest, «First Nations — Royal Collections", loc. cit., 53 ; J. Lainey, La "monnaie des Sauvages... », op. cit., 70.

34. Il s'agit sans doute de Louis-Léon Pajot, comte d'Ons-en-Bray (Onsenbray) né le 25 mars 1673 à Paris et décédé le 2 février 1754 à Bercy. Il fut intendant général des postes et relais de France, et membre honoraire de l'Académie royale des sciences. Il établit son laboratoire et cabinet d'histoire naturelle dans sa maison à Bercy où il y fit des recherches en histoire naturelle et en mécanique tout en y amassant une immense collection qu'il lèguera ensuite à l'Académie, en 1753 ; Annie Charon, dir., "Catalogue des livres et estampes de la bibliothèque de feu Monsieur Pajot, comte d'Onsenbray, honoraire de l'Académie royale des Sciences ». Esprit des livres, catalogues de vente de bibliothèques de l'époque moderne conservés dans les bibliothèques parisiennes (Paris, Éditions École nationale des chartres, 2002), Éditions en ligne de l’École des chartres, $n^{\circ} 2$, http://elec.enc.sorbonne.fr/cataloguevente/notice471.php (consulté le 11 juillet 2008).

35. C. F. Feest, dir., Premières nations, collections royales..., op. cit.

36. Ibid., 46. 
observer, objets qui, comme plusieurs autres issus de nombreux cabinets privés, ont fini leur course au musée du quai Branly. Ces objets sont-ils ceux que nous pouvons observer aujourd'hui? Il est heureusement possible de le croire.

\section{LE DÉVELOPPEMENT D'UNE INTERPRÉTATION ERRONÉE}

Étant donné l'absence de renseignements fiables et vérifiables sur le prétendu wampum que les Hurons auraient offert à Champlain, et comme la diffusion d'une interprétation douteuse continue d'avoir cours à l'heure actuelle, on peut se demander d'où elle provient, comment elle s'est constituée, quand elle est apparue et aussi comment se fait-il que plusieurs chercheurs la répètent depuis sans la vérifier.

Selon nos recherches, la première mention d'une partie de cette interprétation provient de Ernest-Théodore Hamy, conservateur au musée du Trocadéro, qui deviendra le Musée de l'Homme, dont une partie des collections forme aujourd'hui le musée du quai Branly. Dans un texte présenté à la Société des américanistes de Paris vers 1895-1896, il devient le premier à suggérer que ce wampum représente les "Quatre Nations huronnes ${ }^{37}$ ». Après avoir expliqué rapidement la constitution de la société huronne, il affirme:

C'est cette confédération, représentée par quatre Indiens tirant de l'arc, qui apparaît sur ce monument tout à fait unique que j'ai l'honneur de présenter à la Société. Ce petit monument n'est autre qu'une de ces ceintures en wampum qui jouaient jadis un rôle si important dans les relations des tribus indiennes, soit entre elles, soit avec les Blancs ${ }^{38}$.

On admettra qu'une telle attribution est très rapide et plutôt arbitraire. Pourquoi les Hurons? Sans doute parce que ceux-ci étaient bien connus des Français pour avoir été leurs alliés depuis longtemps. Hamy commence d'ailleurs son article ainsi: «Les Indiens du Canada, fidèles alliés des Français, que nous connaissons sous le sobriquet de Hurons ${ }^{39}$...». L'auteur conclut en suggérant un moment historique pendant lequel ce wampum put être échangé:

37. Dans son ouvrage sur les origines du Musée du Trocadéro publié en 1889, Hamy ne glisse pas un mot sur ce wampum en particulier, et ce, bien qu'il fasse l'historique des collections ethnographiques de ce musée; E.-T. Hamy, Les Origines du Musée d'Ethnographie..., op. cit., chap. III.

38. E.-T. Hamy, «Note sur un wampum représentant les quatre-nations... », loc. cit., 164.

39. Ibid., 163. 
Le wampum où sont représentées les Quatre-Nations huronnes dut être présenté dans des conditions particulièrement importantes. [...] Peut-être est-ce un des colliers qui furent échangés lorsque, dans des circonstances particulièrement solennelles, Frontenac négocia la paix entre les Iroquois et les Hurons en juillet $1673^{40}$.

Encore une fois, rien ne permet d'appuyer sérieusement cette interprétation, et celle-ci devient même plutôt farfelue si l'on sait qu'en 1673, la confédération huronne qui regroupait quatre nations (certains disent cinq) est dispersée depuis environ vingt ans. Pour l'instant, notons que Champlain n'est nullement mentionné et que l'année suivante, Hamy attribue toujours ce wampum aux quatre nations huronnes sans ajouter d'autres informations pouvant appuyer une telle hypothèse ${ }^{41}$.

Sensiblement à la même époque, alors qu'il prépare son étude magistrale sur les wampums (désormais un incontournable), William M. Beauchamp eut vent de l'existence des wampums du Trocadéro par l'intermédiaire d'un dénommé W. L. Hildburgh, alors en Europe. À partir des photographies de son informateur, Beauchamp décrit brièvement l'objet qui nous intéresse sans mentionner l'interprétation de Hamy qui, visiblement, n'était pas encore connue en Amérique ${ }^{42}$. Le wampum apparaît par la suite dans la publication de René Thévenin et Paul Coze intitulée Mœurs et Histoire des Indiens Peaux-Rouges parue en 1928 à Paris. L'explication fournie, très brève, se lit comme suit: "Ceinture Huron - en wampums, représentant quatre Indiens tenant leurs arcs - gris et blancs ${ }^{43}$ ».

Le wampum est ensuite présenté lors de l' "Exposition rétrospective des colonies françaises de l'Amérique du Nord» qui eut lieu à Paris en 1929. Arthur G. Doughty et Gustave Lanctot des "Archives du Canada» font alors partie du «comité du Canada». Le wampum est ainsi présenté: "Ceinture en Wampums. xviII siècle. Ceinture en perles wampums. Quatre Indiens tenant leurs arcs. Ceinture huronne ${ }^{44}$ ». Notons que la mention de l'origine huronne est conservée, que celle à Champlain n'apparaît toujours pas et que la date est repoussée au $\mathrm{XVIII}^{\mathrm{e}}$ siècle.

40. Ibid., 166.

41. E.-T. Hamy, Galerie américaine du Musée d'ethnographie du Trocadéro : choix de pièces archéologiques et ethnographiques (Paris, Ernest Leroux, 1897), 1.

42. W. M. Beauchamp, Wampum and Shell Articles..., op. cit., 425, 476, pl. 25, fig. 274.

43. René Thévenin et Paul Coze, Mœurs et Histoire des Indiens Peaux-Rouges (Paris, Payot, 1928), 329.

44. L. Leymarie, Catalogue illustré..., op. cit., 4 et fig. 2. 
Quelques années plus tard, soit au milieu des années 1950, Marius Barbeau fait la tournée des musées européens afin d'y identifier les objets et collections d'intérêt nord-américain. Accompagné de George-Henri Rivière, Barbeau notera à propos du même wampum: "ceinture des 4 chasseurs à l'arc, vient du Canada, origine inconnue. 3 pieds de long ${ }^{45}$ ». Le lien avec Champlain? Barbeau n'en souffle mot. Pas plus d'ailleurs dans le compte rendu de ses visites publié l'année suivante ${ }^{46}$.

En somme, seuls les auteurs européens que sont Hamy, Leymarie, Thévenin et Coze associent l'objet aux Hurons, alors que Beauchamp et Barbeau, chercheurs américain et canadien respectivement, admettent que l'origine est inconnue.

Ainsi donc l'information récurrente concernant ce wampum était qu'il provenait des Hurons, sans plus. Et pourtant, par la suite et jusqu'à tout récemment, des informations se sont ajoutées. On indique maintenant qu’il aurait été donné à Champlain, on précise l'année, 1611, pour enfin fixer le nom de celui qui l'aurait offert, Atironta, chef des Arendaronnons (une des quatre nations huronnes), qui devint par la suite le principal allié des Français et le porte-parole des Hurons lors des négociations futures.

Il est difficile de savoir exactement à quel moment les informations supplémentaires s'ajoutent à l'interprétation émise initialement par le conservateur Hamy en 1895-1896, mais elles pourraient bien être issues de l'initiative d'Anne Vitart-Fardoulis, conservatrice au département de l'Amérique du Nord du Musée de l'Homme de Paris. En effet, dans le catalogue de l'exposition Le Canada de Louis XIV organisée par la ville de Saint-Germain-en-Laye avec le concours de l'Ambassade du Canada et de la Fondation MacDonald Stewart de Montréal, qui eut lieu dans la ville de Saint-Germain-en-Laye du 10 octobre au 15 novembre 1980, Anne Vitart-Fardoulis présente, pour ce qui semble être la première fois, une interprétation étonnamment exhaustive du wampum qui nous intéresse. Citons les passages les plus pertinents à notre propos:

Wampum des quatre nations hurones [sic] donné à Champlain en gage d'alliance contre les Iroquois. Cette alliance était toujours en vigueur à l'époque de Frontenac. [...] Le wampum présenté ici, dit «des Quatre Nations Hurones» [sic] a très probablement été échangé aux environs de 1611 entre Champlain et les Hurons qui demandaient, par l'intermédiaire de ce document, l'alliance avec la France. Effectivement, deux ans après l'expédition de Champlain

45. Musée canadien des civilisations, Fonds Marius Barbeau, "Collection nord-américaine, Trocadéro, Paris », boîte (temporaire) 200, dossiers 31 et 32.

46. Marius Barbeau, "Early Americana », Canadian Geographical Journal, 53,1 (1956): 2-11. 
contre les Mohawks (groupe iroquois), le conseil des chefs de la Confédération hurone $[s i c]$ envoya en secret à Champlain un présent de grande valeur, en lui demandant de conclure un traité d'alliance avec eux. Il semble donc que ce wampum ait été la "voix» de Atironta, chef des Arendaronons, un des quatre groupes qui formaient à ce moment, la confédération hurone [sic]. En effet, dès 1630, les annales Jésuites parlent des cinq tribus de la confédération. Or le wampum présenté ici ne montre que quatre silhouettes humaines ce qui semble prouver que ce document a été échangé avant 1630, alors que les Hurons n'étaient que quatre groupes à former la confédération. Il semble que le Wampum des Quatre Nations ait été rapporté en France par Frontenac en 1682. Indiens Hurons. Canada. $83 \times 12 \mathrm{~cm}^{47}$.

L'analyse et les explications sont extrêmement rapides pour qu'on lui accorde une réelle crédibilité d'autant plus qu'aucune source n'appuie ces prétentions. Dans au moins deux autres publications, la conservatrice au Département de l'Amérique du Nord du Musée de l'Homme reprend cette interprétation sans pour autant l'appuyer par des documents ou témoignages précis ${ }^{48}$. Par la suite, d'autres publications, souvent adressées à un large public, ont repris cette hypothèse avec la même assurance, ce qui contribua à la cristalliser définitivement. De fait, des recherches et expositions récentes, et sérieuses, ont repris ces informations, sans les remettre en question, sans doute en raison de leur importance et de leur sens impressionnant ${ }^{49}$. Il est possible que cette information qui semble avoir été mise en œuvre par Vitart-Fardoulis ait effectivement été fournie

47. Anne Vitart-Fardoulis [sa contribution dans], Le Canada de Louis XIV (Ville de SaintGermain-en-Laye, Musée des Antiquités nationales, 1980), 112-113.

48. Anne Vitart-Fardoulis, «Les objets américains de l'Hôtel de Sérent ou une collection ethnographique au $18^{e}$ siècle", Archivio per l'Antropologia e la Etnologia, CXIII (1983): 146; Anne Vitart-Fardoulis, "Chronique d'une rencontre en terre de Canada», dans Daniel Lévine, dir., Amérique, continent imprévu. Le rencontre de deux mondes (Paris, Bordas, 1992), 98.

49. Thomas H. Flaherty, dir., Realm of the Iroquois (Alexandria, Virginia, TimeLife Books, 1993), 73; Michel Lessard, avec la collaboration de Pierre Lahoud, L'Île d'Orléans: aux sources du peuple québécois et de l'Amérique française (Montréal, Éditions de l'Homme, 1998), 128; Recherches amérindiennes au Québec, 30,1 (2000): page couverture et page 1; Alain Beaulieu et Roland Viau, La Grande Paix: chronique d'une saga diplomatique (Montréal, Libre Expression, 2001), 51; Peter Cook, «Vivre comme frères. Le rôle du registre fraternel dans les premières alliances franco-amérindiennes au Canada (1580-1650)", Recherches amérindiennes au Québec, 31,2 (2001): 56; Gilles Proulx, "Québec", dans James C. Kelly et Barbara Clark Smith, Jamestown - Québec — Santa Fe: Three North American Beginnings (Washington/New York, Smithsonian Books, 2007), 100. Notons enfin qu'une reproduction de ce wampum se trouve à la Maison de l'émigration française au Canada de Tourouvre (Orne, France). La notice descriptive mentionne la même interprétation (Champlain, 1611, etc.) et l'attribue faussement à la collection de Parcs Canada. Après quelques vérifications, et au hasard d'une discussion, nous apprenions qu'il s'avère avoir été produit sur commande par un ami et artisan huron-wendat de Wendake, Michel Savard. Nos remerciements à Julie Rodrigue pour nous avoir mentionné l'existence de cette reproduction de wampum. 
et diffusée par le musée détenteur du wampum en question. En fonction de quelle information nous l'ignorons mais, nous l'avons vu, les catalogues antérieurs sont pourtant muets à cet égard.

Ce genre de glissement s'opère probablement lorsque les historiens et autres chercheurs qui pigent dans les collections d'objets historiques et patrimoniaux pour illustrer ou expliquer leurs propos recueillent de l'information qui n'est pas toujours exacte et vérifiée. Les conservateurs ou les responsables d'un musée peuvent présenter au chercheur l'objet et l'information qui l'accompagne de façon imprudente, et ce dernier tient ensuite pour acquis que cette information est fiable. Pis encore, parce que plus simple et plus rapide, l'information erronée peut être reprise d'une publication à une autre. Plusieurs erreurs d'interprétation ont ainsi été diffusées.

Quoiqu'il en soit, désormais cette information circule, constamment reprise mais jamais vérifiée. Ainsi, d'une vague évocation, l'on passe à une affirmation qui devient elle-même une nouvelle vérité. On ne sait pas d'où elle vient, mais on la reprend, parce qu'elle nous convient, mais aussi parce qu'on a tendance à croire sur parole, sans oser remettre en question, ce que dit une si prestigieuse institution. Si le Musée de l'Homme de Paris dit quelque chose, cela doit bien être vrai! Les trésors royaux de France imposent le respect.

\section{LA SACRALISATION DES OBJETS MÉMORIELS}

Ce qui étonne peut-être davantage, à notre avis, ce n'est pas tant la facilité avec laquelle la conservatrice du Musée de l'Homme est en mesure d'affirmer de tels propos, mais plutôt l'aisance avec laquelle les chercheurs les acceptèrent et les répétèrent par la suite. Si le fait que des auteurs d'ouvrages importants aient si facilement tombé dans le piège tient en outre au fait que les colliers de wampum étaient jusqu'à récemment très peu documentés dans l'historiographie, cela est sans doute aussi dû au fait que ce lien à Champlain répond à un besoin de comprendre, d'expliquer, d'identifier, de concrétiser le passé, de s'en approcher.

À ce sujet, les réflexions de Jean-Pierre Chrestien, conservateur au Musée canadien des civilisations, à propos de l'astrolabe dit de Champlain conservé à cet endroit sont intéressantes à maints égards ${ }^{50}$. Interviewé par

50. Ce fameux astrolabe a été trouvé par le jeune Edward George Lee au mois d'août 1867 sur la rive du lac Green en Ontario. Le doute persiste toujours quant à son origine véritable. Pour un aperçu du débat qui l'entoure, voir Jean-Pierre Chrestien, "L'astrolabe dit de Champlain», dans Raymonde Litalien et Denis Vaugeois, dir., Champlain. La naissance de l'Amérique française 
le journaliste Paul Gessel dans le cadre d'une série d'articles entourant le $150^{\mathrm{e}}$ anniversaire du Musée, monsieur Chrestien affirmait que le public souhaitait que Champlain ait effectivement possédé cet astrolabe, astrolabe par ailleurs qui serait l'un des plus importants artefacts issus de la Nouvelle-France et certainement le plus important objet relié à Champlain. Selon lui, le public a besoin de ce genre de point de repère historique: "we need those things to build our history. If we have nothing to relate with in the past, then it's something a little artificial. It's fantastic to be able to touch it and say, "This was used by Champlain" ${ }^{51}$.» Ainsi, toucher l'objet reviendrait un peu à toucher Champlain lui-même et à se transporter, le temps d'un instant, au tout début du XVII siècle en train de canoter sur la rivière des Outaouais. Il est bien vrai que «le culte des héros a besoin de reliques», comme l'a si bien dit l'historien Patrice Groulx ${ }^{52}$.

Ainsi sacralisés, les objets deviennent les véhicules des représentations et des discours relatifs à la mémoire et à l'identité collectives. Justement, le wampum dit de Champlain relie deux éléments bien utiles à la définition de cette identité : il implique un "héros», Champlain, et un événement d'importance historique majeure, soit la formalisation d'une alliance qui allait assurer les débuts de l'implantation française en Amérique ${ }^{53}$. À partir du moment où un sens particulier lui est donné, l'objet est beaucoup plus qu'un simple artefact ethnographique. Sa nouvelle signification et ce à quoi il réfère désormais transcendent de loin sa simple fonction utilitaire d'origine. Ce wampum n'est plus seulement un exemplaire des quelque deux ou trois cents wampums connus de par le monde, mais il devient l'un des plus importants, des plus anciens et des plus significatifs qui soient. Dans cette optique, il est clair que les traces du passé servent parfois davantage de support au discours identitaire actuel que d'outils pour documenter l'histoire. Jacques Lacoursière et Jacques Mathieu expriment parfaitement bien ce phénomène de glorification des objets lorsqu'ils affirment:

(Paris/Sillery, Nouveau Monde/Septentrion, 2004), 351-353; Douglas Hunter, "The Mystery of Champlain's Astrolabe», The Beaver, 84,6 (décembre 2004-janvier 2005): 14-23 ; P. Gessell, "The "Star-Taker" ", loc. cit.

51. Ibid., A6.

52. Patrice Groulx, "Se voir dans la peau de Samuel de Champlain», dans R. Litalien et D. Vaugeois, dir., Champlain..., op. cit., 341.

53. Sur la construction des représentations symboliques du passé, on peut lire des exemples fournis dans le chapitre 7 de l’ouvrage de Jacques Mathieu et Jacques Lacoursière, Les mémoires québécoises (Sainte-Foy, Les Presses de l’Université Laval, 1991). 
L’objet jugé digne de mémoire a le plus souvent perdu sa fonction utilitaire pour acquérir une valeur symbolique et une signification culturelle. Redécouvert après avoir été un temps oublié, l’objet devient témoin d'une époque, d'un espace, d'une culture. Il rend compte des aspirations et des valeurs de la collectivité qui le sacralise, l'admire sans le toucher, le vénère pour ce qu'il signifie plutôt que pour ce qu'il est ${ }^{54}$.

Soulignons enfin que la notoriété d'un objet ne contribue pas seulement à augmenter sa valeur symbolique: sa valeur monétaire s'accroît tout autant. Douglas Hunter nous apprend qu'en 1989, le gouvernement fédéral accepta de débourser un énorme montant de 250000 \$ pour le présumé astrolabe de Champlain, alors entre les mains de la New York Historical Society. Ce qui est intéressant de constater, c'est qu'un tel objet, n'eût été de sa provenance hypothétique et non résolue, aurait pu être acquis pour un montant moindre allant de $50000 \$$ à $100000 \$ 55$.

\section{DISCUSSION AUTOUR DE LA NOUVELLE INTERPRÉTATION PROPOSÉE}

Il importe de souligner que malgré sa popularité, l’idée que le wampum du musée du quai Branly ait été offert à Champlain a tout récemment été mise en doute. Dans le catalogue de l'exposition «Premières Nations Collections Royales» produit par le musée du quai Branly, l’auteur Christian Feest précise:

On l'a longtemps crue d'origine huronne et en relation avec les quatre tribus huronnes du début du xviI siècle. Cependant, sur la base de considérations stylistiques, il s'agirait plus probablement d'une ceinture provenant de la région des Grands Lacs occidentaux, et serait peut-être liée au quatre «clans» ottawa. $[\ldots]$ xviII ${ }^{\mathrm{e}}$ siècle $^{56}$.

Dans ses deux publications portant sur les collections royales de France, Feest se base sur des «considérations stylistiques» pour documenter ce

54. J. Mathieu et J. Lacoursière, Les mémoires québécoises, op. cit., 344.

55. D. Hunter, "The Mystery of Champlain's Astrolabe», loc. cit., 15.

56. C. F. Feest, dir., Premières nations, collections royales..., op. cit., 18, 73. Dans un article publié dans le American Indian Art Magazine, Feest nuance en affirmant: «the belt is more likely to be of Algonquian (perhaps Ottawa) manufacture and was probably made in the eighteenth century"; C. F. Feest, «First Nations - Royal Collections», loc. cit., 52. Sur les quatre "clans» outaouais, voir Johanna E. Feest et Christina F. Feest, "Ottawa», dans William C. Sturtevant, dir., Handbook of North American Indians, vol. 15 ; Bruce G. Trigger, dir., Northeast (Washington, D. C., Smithsonian Institution, 1978), 772-786. Ruth Phillips avait déjà remis en question cette interprétation lors de l'exposition présentée au Glenbow Museum de Calgary en 1988 ; voir Julia D. Harrison, The Spirit Sings. Artistic Traditions of Canada's First Peoples. A Catalogue of the Exhibition, (McClelland and Stewart, Glenbow Museum, 1987), 47. 
wampum sans pour autant expliquer au lecteur ce que cela signifie réellement. Ce n'est que dans une autre publication portant sur les collections amérindiennes conservées au musée d'Yverdon en Suisse que Feest élabore sur ce qu'il entend par l'expression "considérations stylistiques », en insistant sur deux caractéristiques particulières à ce wampum : son orientation verticale (les personnages s'observent de haut en bas, plutôt que d'être côte à côte comme c'est généralement le cas des wampums comportant des silhouettes humaines), et la forme et couleur des figures représentées (le tronc du corps est de forme rectangle plutôt que conique, et les silhouettes sont blanches sur fond pourpre $)^{57}$.

Bien que la période proposée soit probable, nous croyons que cette nouvelle attribution (Outaouais), bien qu'elle soit possible elle aussi, n'est pas plus crédible du fait qu'elle n'est pas davantage vérifiable. Poussons alors un peu plus loin la réflexion et permettons-nous de douter de l'argument principal utilisé par Feest. Bien entendu, le but de l'exercice n'est nullement de discréditer les importants travaux de Christian Feest mais plutôt de discuter des possibilités et des dangers de vouloir proposer à tout prix une interprétation, une date et une origine aux objets aujourd'hui dans les musées ${ }^{58}$. Une des principales faiblesses de cette nouvelle interprétation est qu'elle compare le wampum du musée du quai Branly avec trois autres objets qui ne sont pas des wampums, soit deux bandes perlées (l'une conservée au Musée d'Yverdon et l'autre faisant partie de la King's Regiment Collection conservée au National Museums Liverpool en Angleterre), dont la fonction n'est pas tout à fait déterminée, et une parure comportant des perles de verre devant être portée autour du cou conservée au Saffron Waldon Museum d'Angleterre.

La bande perlée du musée d'Yverdon, que Feest décrit comme un "glass imitation wampum belt» ou encore de «belt of glass imitation wampum», comprend bien six silhouettes blanches avec le tronc rectangle ${ }^{59}$. Mais elle est faite de perles de verre, et non de wampum; voilà toute une différence!

57. C. F. Feest, «Early Woodlands Material in the Musée d’Yverdon», dans J. C. H. King et C. F. Feest, dir., Three Centuries of Woodlands Indian Art: A Collection of Essays (Altenstadt, ZKF Publishers, ERNAS Monographs 3, 2007), 18.

58. À noter que la nouvelle interprétation de Feest semble avoir été acceptée par les conservateurs. En effet, c'est celle-ci qui a été retenue pour l'exposition «Jamestown, Québec, Santa Fe - Trois berceaux nord-américains" présentée au Musée canadien des civilisations du 9 mai au 7 septembre 2008. Au départ, c'est pourtant le lien à Champlain qui prévalait, d'où l'insertion de cet objet dans l'exposition destinée à commémorer la fondation de Québec, voir G. Proulx, "Quebec», loc. cit., 100.

59. Trois silhouettes sont à l'endroit et trois sont à l'envers. Ainsi, ce n'est que lorsque le «wampum» est suspendu par le milieu que les six figures sont toutes à l’endroit. 
Tous les objets perlés de facture amérindienne ne sont pas des wampums et d'ailleurs toutes les perles faites de coquillages marins ne sont pas du wampum, et ce, malgré ce que plusieurs notices descriptives des musées peuvent en dire. Pourtant, certains chercheurs affirment qu'à défaut d'avoir accès aisément à du wampum, les groupes éloignés des sources de perles, comme ceux habitant le sud des Grands Lacs (Lower Great-Lakes) offraient des colliers en perles de verre lors des rencontres diplomatiques, dès la fin du XviI ${ }^{\mathrm{e}}$ siècle $^{60}$.

Ce n'est pourtant pas ce que les archives coloniales nous disent: advenant un manque de wampum, de simples branches de wampum ou même d'autres objets, tels des bâtonnets, étaient donnés temporairement, jusqu'à ce que de véritables colliers de wampum les remplacent ${ }^{61}$. En Nouvelle-France où le wampum fit parfois cruellement défaut (notamment vers 1710-1730), on tenta même de fabriquer des perles à partir d'autres matériaux, tel le marbre; mais ce fut en vain, car seul le wampum convenait ${ }^{62}$. Puisqu'aucune preuve documentaire n'a été fournie jusqu’à maintenant pour soutenir cette assertion voulant que des colliers de perles de verre aient circulé de la même façon que des colliers de wampum, il demeure pratiquement certain que seuls les colliers faits de véritables perles de wampum furent utilisés dans les contextes politiques et diplomatiques que l'on connait.

En ce sens, l'objet conservé au musée d'Yverdon pourrait n'être qu'une sangle décorative servant à porter un sac, une corne à poudre ou n'être qu'une bande perlée dont la fonction serait purement esthétique. Il existe en effet de ces sangles qui reprennent pratiquement les mêmes motifs que l'on retrouve sur les colliers de wampum ${ }^{63}$. Dans ce cas-ci, le fait que les

60. Communication de George Hamell à Marshall J. Becker, 23 mai 2007.

61. W. M. Beauchamp, Wampum and Shell Articles..., op. cit., 455. Nous avons donné des exemples pour le $\mathrm{XVII}^{\mathrm{e}}$ siècle à la note 12 . On peut lire d'autres exemples plus tardifs dans «Report of Treaty at Logstown", 29 mai 1753, dans F. Jennings, dir., Iroquois Indians : A Documentary History..., op. cit., bobine 15; et dans «Letter from Governor Hunter to English Board of Trade and Plantations concerning results of recent interviews with the Five Nations in August, with enclosures », 29 septembre 1715, dans Ibid., bobine 8. Ces deux exemples sont cités dans J. Lainey, La "monnaie des Sauvages"..., op. cit., 46, 52-53.

62. Ibid., 12-14, 25.

63. Un bel exemple est illustré dans R. B. Phillips, «L'art dans la vie des peuples des terres boisées: le début de la période des explorateurs ", dans Michel Desrosiers et al., dir., Le Souffle de l'esprit. Coutumes et traditions chez les Indiens d'Amérique (Montréal/Paris, Éditions Québec/ Amérique/Éditions Jean Piccolec, 1988), 85, fig. 78, et dans C. F. Feest, "German Collections from the American Revolution", dans J. C. H. King et C. F. Feest, dir., Three Centuries of Woodlands Indian Art..., op. cit., 52, note 54, fig. 13. La sangle du sac de "style iroquois» a six rangs de large et est illustrée de sept paires de petits losanges, et de trois lignes à chaque extrémité. Même chose 
figures soient tête-bêche (trois vers le haut, trois vers le bas) expliquerait cette fonction: lorsqu'elle est utilisée à cette fin, la bande perlée montre les six personnages à l'endroit. Notons qu'elle mesure $104 \mathrm{~cm}$, ce qui représente une taille pratique, voire idéale pour cette fonction.

Pour ce qui est de la seconde bande perlée, celle conservée en Angleterre sur laquelle sont illustrées douze figures humaines avec sensiblement les mêmes caractéristiques, elle est aussi en perles de verre mais sa taille gigantesque (avec ses 31 rangs de large, elle contient peut-être plus de 12000 perles) fait en sorte qu'elle n'a probablement jamais eu la même fonction utilitaire. Si effectivement cet objet d'envergure fut offert par les Outaouais à Arent Schuyler DePeyster, gouverneur à Détroit, comme il a été suggéré ${ }^{64}$, il devrait normalement être mentionné et brièvement décrit dans les archives, c'est-à-dire dans le procès-verbal ou le compte rendu de cette rencontre. Encore une fois, aucune preuve documentaire prouvant cette assertion n'a été fournie à ce jour, ce qui tend davantage à confirmer notre hypothèse: les "wampums» de verre n'ont jamais remplacé les colliers de wampum dans les contextes diplomatiques et politiques.

En ce qui a trait au troisième objet comparé, soit la très belle parure devant être portée autour du cou, elle renferme effectivement une silhouette humaine blanche sur fond noir avec un tronc de forme rectangle, et serait de «style outaouais ou wyandot ${ }^{65}$ ». Mais, nous le répétons, il s'agit là d'un objet n'ayant que peu à voir avec les colliers de wampum, sinon qu'il comporte une silhouette humaine perlée lui aussi. Il est sans aucun doute utile de comparer les styles dans l'analyse de la culture matérielle autochtone (vases, sacs, ornements, etc.), mais dans l'étude des colliers de wampum, ces outils diplomatiques ayant une tout autre fonc-

pour une bande perlée conservée au Musée de la civilisation de Québec (nº 1992-1292): elle mesure $104 \mathrm{~cm}$ et comporte des motifs similaires à ceux que l'on retrouve sur les wampums; voir J. Lainey, La "monnaie des Sauvages...», op. cit., 177-179, 272, $3^{\text {e }}$ de couverture. Voir aussi le sac soutenu par une sangle faite de perles vertes et jaunes illustré dans G. Proulx, "Québec», loc. cit., 101, et celui illustré dans T. H. Flaherty, dir., Realm of the Iroquois..., op. cit., 147. Enfin, notons qu'un sac du genre est conservé au Musée d'histoire naturelle et d'ethnographie de Lille, no d'inventaire 990.2.3316, et qu'un autre superbe exemplaire peut s'observer au Peabody Museum de Harvard.

64. Simon Jones, "Caldwell and DePeyster: Two Collectors from the King's Regiment on the Great Lakes in the 1770s and 1780s», dans J. C. H. King et C. F. Feest, dir., Three Centuries of Woodlands Indian Art..., op. cit., 33, fig. 2; David A. Armour et Keith R. Widder, At the Crossroads. Michillimackinac During the American Revolution (Mackinac Island, Mackinac State Historic Parks, 1978), 20, 24, 77, 107.

65. R. B. Phillips, «L'art dans la vie des peuples des terres boisées...», loc. cit., 89, fig. 82. 
tion, cette approche ne s'avère guère pertinente, d'autant plus qu'elle ne semble pas avoir été retenue dans la littérature sur les wampums.

Le danger d'une telle approche comparative est qu'elle suggère désormais qu'un wampum comportant des silhouettes humaines blanches avec un tronc rectangle agencées de haut en bas plutôt que côte à côte serait d'origine algonquienne, possiblement outaouaise. En affirmant qu'un wampum ayant ces caractéristiques particulières soit de «style» outaouais, on avance que d'autres wampums ayant des figures humaines similaires et se présentant à la verticale soient aussi outaouais, ce qui est un terrain glissant. Pour cause, des wampums ayant justement ces caractéristiques ont été collectionnés auprès des Iroquois et n’auraient pas impliqué les Outaouais. C'est le cas entre autres du «Remembrance Belt», acquis du conseil d'Onondaga en 1898, qui se «lit» à la verticale et dont le tronc de l'individu illustré est de forme rectangulaire ${ }^{66}$. Les individus sur le «Wolf Belt», acquis à Akwesasne en 1898, ont aussi tous deux le tronc rectangulaire ${ }^{67}$, tout comme c'est le cas pour ceux représentés sur le «Penn Wampum Belt ${ }^{68}$ ». Ces wampums seraient-ils, de fait, aussi de "style outaouais »? Et qu'en est-il des wampums avec des losanges? ils sont iroquois? hurons? algonquins? et ceux avec des hexagones?

En fait, il est clair que les motifs que l'on retrouve sur les wampums sont trop restreints en nombre pour servir à distinguer l'origine ethnique des colliers. De plus, on sait maintenant que pratiquement n'importe qui pouvait fabriquer des colliers de wampum; un «style» national est donc peu probable ${ }^{69}$. Ce questionnement nous amène vers une autre interrogation constante lorsqu'on tentera de déterminer l'origine des colliers que l'on étudie: qui détermine l'origine ethnique d'un wampum, le fabricant ou le dépositaire? Les wampums étant généralement reconnus comme des objets que l'on offre, ceux que l'on retrouve dans une communauté donnée ont donc été offerts à celle-ci; ils n'émanent pas nécessairement de cette communauté. Enfin, si cette nouvelle hypothèse sur l'origine de ce wampum était vraie, cela serait davantage un coup de chance. En précisant «Outaouais», "Grands Lacs occidentaux» et «XVIII

66. Noah. T. Clarke, "The Wampum Belt Collection of the New York State Museum», New York State Museum Bulletin, 288 (1931): 95-96, 114, 115, fig. 28; W. M. Beauchamp, Wampum and Shell Articles..., op. cit., 413-414, 422, pl. 20, fig. 237.

67. N. T. Clarke, "The Wampum Belt Collection...", loc. cit., 110, 119, fig. 37 ; W. M. Beauchamp, Wampum and Shell Articles..., op. cit., 427, pl. 19, fig. 229.

68 Ibid., 393-394, pl. 13, fig. 173 ; F. G. Speck et W. C. Orchard, The Penn Wampum Belts..., op. cit.

69. J. Lainey, La «monnaie des Sauvages»..., op. cit., 30-31, 76-80, 164, 178, 181, 202-203. 
siècle» dans la description du wampum, on demeure extrêmement prudent et on s'assure de miser juste, surtout si l'on connaît la mobilité des Outaouais qui vécurent auprès d'autres groupes, parfois dans les mêmes établissements, à plusieurs endroits, au cours du XviI ${ }^{\mathrm{e}}$ siècle $^{70}$.

\section{CONCLUSION}

L'année 2008 est celle du $400^{\mathrm{e}}$ anniversaire de la fondation de la ville de Québec, entre autres, par Champlain. Nous sommes d'avis que l'heure des commémorations est aussi un bon moment pour faire certaines rectifications et mises au point. Différents objets, lieux, personnages et événements ont souvent servi de façon quelque peu émotive à construire un discours identitaire, mais l'objectivité des données historiques et le recul nous font voir les choses bien autrement. Bien sûr nos arguments pris un à un isolément sont insuffisants pour documenter avec précision le wampum ici étudié. Ce n'est qu'en les additionnant et les superposant que l'évidence saute aux yeux: ce wampum n'est pas celui que reçut Champlain en 1611. Il est bien vrai aussi qu'il est beaucoup plus facile de dire ce qu'un objet n'est pas que d'expliquer ce qu'il est ou ce qu'il était. Ce silence qui entoure l'objet est beaucoup moins intéressant, d'un point de vue muséologique, qu'une interprétation frappante comme celle impliquant Champlain.

L'observateur aimerait croire que ce dernier l'a manipulé après l'avoir reçu des Hurons afin de forger une alliance qui allait garantir le succès du développement de la Nouvelle-France. N'a-t-on pas voulu que Champlain soit le héros fondateur des Québécois? N’a-t-il pas été considéré par plusieurs historiens comme l'un des personnages les plus importants de l'histoire du Québec et du Canada, comme le "père de la NouvelleFrance»? Tout en augmentant considérablement la valeur des collections, posséder un témoin de cette «fondation» ferait en sorte que le lointain et abstrait passé se rapproche de nous et se concrétise quelque peu, par l'objet matériel. Pourtant, tout porte à croire que Champlain n'a jamais vu ni touché le wampum du musée du quai Branly.

Les musées qui préservent et font connaître notre patrimoine matériel ont entre autres comme fonction de contribuer au développement d'une identité nationale ${ }^{71}$. Si cette identité a besoin de se projeter dans l'avenir

70. J. E. Feest et C. F. Feest, «Ottawa», loc. cit. ; Helen Hornbeck Tanner, dir., Atlas of Great Lakes Indian History (Norman, University of Oklahoma Press, 1987).

71. De nombreux ouvrages ont abordé cette thématique. La première partie de l'article de Philippe Dubé et Andrée Lapointe offre plusieurs références intéressantes: «Le musée en tant 
pour se forger, il est clair qu'elle nécessite aussi un retour dans le passé et qu'elle a besoin de points de repère, de lieux ou d'objets phares pour se situer. Or, comme nous le rappellent Jacques Lacoursière et Jacques Mathieu, le recours à l'histoire comporte toujours une sélection de faits jugés pertinents, ce qui contribue nécessairement à rendre partielle toute évocation du passé qui occulte, ou magnifie, certains faits et événements en particulier en fonction des besoins qui les font ressurgir de l'oubli ${ }^{72}$. C'est ainsi qu'un discours accrocheur impliquant un nom marquant dans l'histoire, Champlain, s'est jumelé à un bien tangible, un collier de porcelaine, objet qui devint dès lors une borne temporelle et matérielle à laquelle on pouvait se rattacher.

Mais pourquoi, pourrait-on s'interroger, importe-il de bien connaitre ces objets particuliers de notre histoire commune? Un élément de réponse serait que les wampums font partie du patrimoine matériel et immatériel autochtone, qu'on y réfère souvent et même de plus en plus. Ils font parfois l'objet de discussions et de débats, ils sont utilisés devant les tribunaux, ou lors de cérémonies, et les communautés autochtones cherchent souvent à les récupérer des mains des musées. Dans ce contexte, il est important de mieux connaître leur histoire et leur origine, pour ainsi mieux les comprendre.

Nous oserions ajouter qu'au-delà des connaissances historiques et muséologiques en général, leur étude est importante du fait que certains individus disent tout simplement n'importe quoi à leur propos. Nous avons vu et entendu des gens affirmer que les wampums étaient des objets qui servaient à traverser les dimensions (cross-dimensions) ou encore que les croix que l'on retrouve sur certains wampums sont plutôt des étoiles, sombrant ainsi dans un mysticisme simpliste et douteux ou évacuant d'emblée toute trace étrangère de chrétienté. Ce n'est qu'en continuant de fouiller les archives de toutes sortes - écrites, orales, iconographiques - et en partageant les résultats de nos recherches que les objets qui nous intéressent ou qui nous concernent pourront être mieux compris.

Traiter d'un objet en particulier est aussi un prétexte pour parler des objets en général, de leur étude et de leur documentation, notamment

que territoire symbolique de la nation", dans Laurier Turgeon, Jocelyn Létourneau et Khadiyatoulah Fall, dir., Les espaces de l'identité (Sainte-Foy, Les Presses de l'Université Laval, 1997), 148-171. Voir aussi l'ouvrage collectif dirigé par Flora E. S. Kaplan, Museums and the Making of "Ourselves": The Role of Objects in National Identity (Londres/New York, Leicester University Press, 1994).

72. J. Mathieu et J. Lacoursière, Les mémoires québécoises, op. cit., 370. 
par les archives qui seules parfois révèlent leur secret. Ainsi, parler du prétendu wampum offert à Champlain nous a permis de voir qu'il est pratiquement impossible de dater les wampums avec précision, que leur attribuer une origine ethnique est pratiquement impensable, que comparer leurs styles s'avère peu utile, que des «wampums" en verre n'ont probablement jamais servi dans les mêmes contextes diplomatiques et que les archives peuvent parfois contrer les profondes lacunes documentaires qui caractérisent les collections anciennes.

L'étude d'un objet en particulier permet aussi de répéter que dans un travail scientifique, les images et les objets doivent être sélectionnés, observés, évalués, contextualisés et les informations s'y rattachant vérifiées tout comme le sont les documents écrits ${ }^{73}$. $S^{\prime}$ interrogeant sur le rôle de l'historien professionnel dans la définition des contenus historiques des musées, Joanne Burgess souligne que jusqu'à tout récemment, l’histoire n'avait que très peu valorisé la culture matérielle et qu'incidemment peu d'historiens-conservateurs avaient pu jouer un rôle dans le développement des expositions et dans l'interprétation des objets présentés ${ }^{74}$. Est-ce par manque d'expertise que l'information erronée sur le wampum dit de Champlain a circulé au moins jusqu'en 2007? C'est possible.

Gardons aussi en tête qu'il faut parfois se méfier de la "tradition orale» des conservateurs et des chercheurs, qui se perpétue, avec ses erreurs d'interprétation, à travers les époques, comme nous l'a rappelé Christian Feest $^{75}$. De même, on ne peut se fier aveuglément aux notices descriptives (anciennes surtout, mais parfois aussi même les plus récentes) des institutions qui les conservent puisque celles-ci ont souvent été rédigées selon le regard de leur époque. En fait, il n’est pas faux de dire que ces informations, qui d'ailleurs brillent souvent par leur absence, sont les témoins de l'histoire des objets, elles sont le résultat de leur collectionnement et de la façon dont on les a traités, tout comme du regard posé sur ces «curiosités» provenant $\mathrm{du}$ "nouveau monde».

73. Personne n'étant à l'abri de telles erreurs, nous avons, bien entendu, nous aussi omis de faire certaines vérifications, notamment pour les figures 5 et 16 dans J. Lainey, La «monnaie des Sauvages"..., op. cit., 44 et 114.

74. Joanne Burgess, "L'historien, le musée et la diffusion de l'histoire», Revue d'histoire de l'Amérique française, 57,1 (été 2003): 37.

75. Caroline Montpetit, «Rencontres d’un autre âge. Montréal accueille les trésors amérindiens du musée du Quai Branly», Le Devoir, 5 juin 2007, A1. 